\title{
SPOTLIGHT EDITORIAL
}

\section{Genomic technologies in leukemia and lymphoma: a review series}

\author{
Leukemia (2009) 23, 1207; doi:10.1038/leu.2009.81
}

The identification and molecular characterization of critical oncogenes and tumor suppressor pathways has dramatically improved our understanding of the molecular basis of leukemias and lymphomas over the last two decades. Most notably, as the number of functional and structural interactions involved in the transformation of hematopoietic progenitors increases we have started to appreciate the interrelationship between different oncogenic lesions leading to the construction of increasingly complex models that represent our current understanding of the molecular circuitry governing cell growth, proliferation and survival in the malignant clone. In this context, the complete sequencing of the human genome represents the most critical step forward towards the elucidation of the molecular networks that control the multistep process of oncogenic transformation in leukemia and lymphoma cells. Instrumentally, the complete sequencing of the human genome has led to the development of high-throughput technologies for the global analysis of gene expression, and protein-protein and protein-DNA interactions. Genome-wide approaches provide a more inclusive view of the molecular circuitries governing the proliferation and survival of malignant cells. As a result, we have gradually changed the framework in which we interpret our experimental data, forcing us to consider the role of each gene in the context of increasingly larger and more complex genetic networks.

The enormous impact of genomic tools in our understanding of the molecular basis of hematopoietic cancers is best exemplified by the way in which microarray gene expression profiling studies have revolutionized our understanding of these malignancies. A decade after the publication of the seminal paper by Golub et al., ${ }^{1}$ describing the ability of gene expression signatures to differentiate between lymphoid and myeloid leukemias, gene expression profiling studies have uncovered the molecular heterogeneity of cancer, defined new prognostic groups, identified new therapeutic targets and established the mechanism of action of numerous oncogenes and tumor suppressors. However, these advances represent only a small fraction of what genomic technologies are to offer in the coming years. Thus, the development of high-throughput sequencing and array platforms to analyze microRNA expression and alternative splicing are already uncovering new levels of transcriptional and translational regulation to be explored. Similarly, large-scale expression profiling studies using cellular perturbations with drugs or RNA interference have started to generate large amounts of dynamic information on the function of genes and pathways. However, the interpretation of genomic data is, in part, limited by the paucity of functional information available for most human genes. As genomic technologies change the way we address questions, analyze data and interpret results, they also generate a new conceptual framework that embraces the complexity, redundancy and dynamic nature of biological systems. We envision that by integrating broad datasets of genetic and epigenetic alterations, gene expression, high-throughput analysis of protein-protein interactions and metabolic profiling, we will overcome the constraints and limitations of our current models to fulfill the promise of rationally designed molecular therapies for the treatment of cancer.

This review series examines the role of genomic tools in the analysis of hematological tumors with an open perspective that includes the review of recent discoveries enabled by these technologies, novel technical breakthroughs and the discussion of future directions, challenges and opportunities in this rapidly evolving and ever exciting field.

\section{Conflict of interest}

The author declares no conflict of interest.

$$
\begin{array}{r}
\text { AA Ferrando } \\
\text { Departments of Pediatrics and Pathology, } \\
\text { Irving Cancer Research Center, } \\
\text { Institute for Cancer Genetics, Columbia University, } \\
\text { New York, NY, USA } \\
\text { E-mail: af2196@Columbia.edu }
\end{array}
$$

\section{Reference}

1 Golub TR, Slonim DK, Tamayo P, Huard C, Gaasenbeek M, Mesirov JP et al. Molecular classification of cancer: class discovery and class prediction by gene expression monitoring. Science 1999; 286: 531-537. 